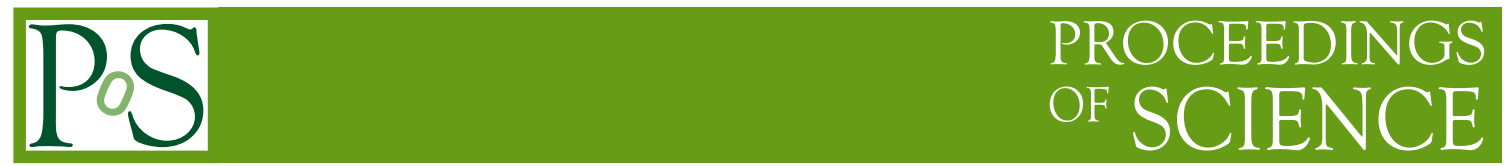

\title{
Directional Dark Matter Search with Nuclear Emulsion
}

\author{
Giuliana Galati* ${ }^{*}$ \\ Istituto Nazionale di Fisica Nucleare \\ E-mail: giuliana.galati@na.infn.it
}

The NEWSdm experiment, based on nuclear emulsions, is proposed to measure the direction of WIMP-induced nuclear recoils. We discuss the potentiality, both in terms of exclusion limits and potential discovery, of a directional experiment based on the use of a solid target made by newly developed nuclear emulsions and read-out systems reaching sub-micrometric resolution.

European Physical Society Conference on High Energy Physics - EPS-HEP2019 -

10-17 July, 2019

Ghent, Belgium

* Speaker.

${ }^{\dagger}$ On behalf of the NEWSdm Collaboration 


\section{Introduction}

Nowadays, compelling evidence suggests that only $15 \%$ of the mass of the Universe is made of ordinary baryonic matter, while the remaining $85 \%$ does neither emit nor absorb any form of electromagnetic radiation, making it practically impossible to be detected through usual astronomical equipments [1]. The Standard Model (SM) of particles and interactions does not include any dark matter candidate. The most compelling DM candidates are Weakly Interacting Massive Particles (WIMPs). Direct detection experiments aim at detecting nuclear recoils produced by the scattering of galactic WIMPs off the nuclei of a target material. The motion of the Solar System through the Galaxy should create an apparent wind of WIMP particles, blowing opposite to the direction of the Sun's motion towards the Cygnus constellation. The intensity of this wind is expected to be time modulated due to the Earth motion in the Solar System, with an annual period and a maximum rate in summer [2]. The DAMA experiment [3] at LNGS has indeed reported a signal with a very clear evidence of annual modulation, as a possible indication of DM induced signal.

The signal rate depends on unknown variables such as the local density and velocity distribution of WIMPs in the Milky Way, the WIMP mass and the interaction cross-section of the target nuclei. In the SM, the speed of WIMPs in the Galaxy is supposed to follow a Maxwellian distribution. However, the signal rate is extremely low, since WIMP's cross-section is smaller than $10^{42} \mathrm{~cm}^{2}$ for nearly all possible WIMP masses, which corresponds to less than 1 detectable event per kg detector mass per year. To detect such a low signal rate, background suppression is fundamental. For this reason, all the direct detection experiments are located in deep underground laboratories, in order to suppress background induced by cosmic rays. The residual background coming from neutrons and gamma rays from the environment and the detector itself can be reduced using an appropriate shielding and high-purity detector components. The ultimate irreducible background comes from solar and atmospheric neutrinos and it is called "neutrino floor". To overcome the neutrino floor, a new generation of detectors is needed, in order to discriminate the neutrino background and identify unambiguously WIMPs as being responsible for the galactic dark matter. These detectors should be capable of measuring the direction of a nuclear recoil track resulting from the elastic scattering of a target nucleus by an incoming WIMP (Weakly Interacting Massive Particle).

From the experimental point of view several approaches have been proposed, for example low pressure gaseous Time Projection Chambers (TPCs) [4]. However, this technology is hardly scalable to very large detectors masses needed to reach a good sensitivity to the Spin-Independent (SI) case. The use of a solid target for directional searches would overcome the mass limitation of gaseous TPC approach thus allowing to reach an high sensitivity in the low cross section sectors of the SI case. Nevertheless, in a solid medium, the track of the WIMP-scattered nuclear recoil will have a path length of the order of a few hundred nanometers, much shorter than in the case of a gaseous target where the recoil length is expected to be of the order of a few millimeters. A detector with high tracking resolution is therefore needed.

\section{The NEWSdm experiment}

NEWSdm is meant to be the first experiment with a solid target for direct directional dark 
matter searches. The approach, proposed by the NEWSdm Collaboration in the Letter of Intent submitted to the LNGS Scientific Committee in 2015 [5], foresees the use of a nuclear emulsionbased detector acting both as target and as tracking device.

The NEWSdm experiment will allow to extend dark matter searches beyond the neutrino floor and provide an unambiguous signature of the detection of Galactic dark matter.

The detector is conceived as a bulk of nuclear emulsions surrounded by a shield to reduce the external background, which will be placed on an equatorial telescope in order to absorb the Earth rotation, thus keeping fixed the detector orientation with respect to the incoming apparent WIMP flux. The angular distribution of the WIMP-scattered nuclei is therefore expected to be strongly anisotropic with a peak centered in the forward direction. To provide an unprecedented position resolution, a new kind of nuclear emulsions, called Nano Imaging Trackers (NIT)[6, 7] has been developed, reaching a granularity one order of magnitude higher than that of the emulsions used in past experiments, such as OPERA[8].

\subsection{NIT: Nano Imaging Trackers}

Nuclear emulsions are a special kind of photographic films which were first used in the early days of cosmic rays studies to detect charged particles, looking at the trace left in the film by the energy released in the emulsion. Nuclear emulsions are made of silver halide crystals embedded in a gelatine matrix. When a charged particle passes through the emulsion medium, the energy released frees silver atoms creating metallic silver on the surface of a bromide crystal, called latent image, not visible yet, made of a few silver atoms on crystals. After the development process, the three-dimensional trajectory of passing through particles can be reconstructed with an optical microscope by connecting consecutively aligned silver grains.

Due to the low recoil energy of a WIMP-scattered nucleus, the expected track length is of the order of a few hundred nanometers. Nuclear emulsion films produced for the OPERA experiment had a linear dimension of the crystals of $200 \mathrm{~nm}$, therefore are not suitable for Dark Matter searches.

For this reason, a novel type of emulsion films, called NIT[6, 7], has been developed, with grain diameters down to a few tens of nm: one order of magnitude smaller than conventional ones, with a linear density of crystals of about 11 crystals $/ \mu \mathrm{m}[6]$.

A key feature of NIT films is the presence, in the emulsion gel, of about ten basic chemical elements. In addition to heavy elements, such as silver and bromine, nuclear emulsions also contain lighter elements, such as Carbon, Nitrogen, Oxygen and Hydrogen. Each one gives a specific contribution to the overall sensitivity, as shown in Fig. ??: indeed, NIT films present a good sensitivity to WIMPs with both light $(5-50 \mathrm{GeV})$ and heavy $(>50 \mathrm{GeV})$ masses, the former will be most effectively be scattered off $\mathrm{H}, \mathrm{C}, \mathrm{N}, \mathrm{O}$, the latter off $\mathrm{Ag}$ and $\mathrm{Br}$.

Thanks to NIT emulsions and to breakthroughs in the development of optical microscopes, it is possible to reconstruct trajectories with path lengths shorter than $100 \mathrm{~nm}$.

\section{Read-out technology}

In the NEWSdm experiment, the expected WIMP signal will consist of nanometric tracks, with an angular distribution peaked in the direction of the Cygnus Constellation, over an isotropically distributed background. 
In order to analyse the target volume over a time scale comparable with the exposure, the scanning system has to be fast and completely automated. Moreover, to ensure high efficiency and purity in the selection of signal candidates, the spatial resolution has to reach the challenging value of a few tens of nanometers, which means an improvement by more than one order of magnitude compared to that achieved with standard optical systems.

The analysis of NIT emulsions is performed with a two-step approach: a fast scanning with a state-of-the-art resolution for the signal preselection, followed by a pin-point check of preselected candidates with unprecedented nanometric resolution to further enhance the signal to noise ratio and perform very accurate measurements of the range and the recoil direction.

In the first phase, a fast scanning, with a speed of almost $200 \mathrm{~cm}^{2} / \mathrm{h}$ [9], is performed by means of an improved version of the optical microscope used for the scanning of the OPERA films $[10,11,12]$. The analysis of the clusters shape helps discriminating between those made of several grains and the ones made of a single grain, which are produced by the thermal excitation (so-called "fog"). Due to the intrinsic resolution of optical microscopes ( 200 nm), two grains in a sequence make a few hundred nanometers long track appear as a single cluster. Compared to a cluster made of a single grain and characterized by spherical shape, clusters made of several grains tend to have an elliptical shape with the major axis parallel to the track direction. Thus, an elliptical fit of the cluster shape can disentangle fog grains from signal tracks.

The directional capability of the elliptical shape analysis has been demonstrated thanks to several tests with ion beams, which were implanted on the emulsion surface at a given direction. First tests were performed in 2012 [13] with $\mathrm{Kr}$ ion beams of 200 and $400 \mathrm{keV}$ kinetic energies. More tests were then carried out with $\mathrm{C}$ ion beams of lower kinetic energies: 60, 80 and $100 \mathrm{keV}$, reaching track lengths in the 100-300 nm range.

To go beyond the optical resolution defined by the Rayleigh criterion, the resonance effect, occurring when nanometric metal grains are dispersed in a dielectric medium, is exploited in the second phase of the measurement [14]. The polarization dependence of the resonance frequencies strongly reflects the shape anisotropy and can be used to infer the presence of non-spherical nanometric silver grains. Images of the same cluster taken with different polarization angles will show a displacement of the position of its barycenter. The analysis of the displacement allows to distinguish clusters made of a single grain from those made of two (or more) grains.

Thanks to this technology is it possible to measure tracks with a length shorter than one hundred nanometers and to achieve an unprecedented resolution of about $6 \mathrm{~nm}$ in both coordinates, as shown in Fig. 1 a), obtained in a test beam with $10 \mathrm{keV} \mathrm{C}$-ions, vertically implanted.

A test has been done with $60 \mathrm{keV}$ Carbon ions. The 2D angular distribution obtained by the direction of the barycentre shift is reported in the figure $1 \mathrm{~b}$ ) for moving and static grains. Clusters not showing any significant displacement of their barycenter represent background-like objects. Moving grains have a clear peak in the direction of the incoming Carbon ions, while static grains show a flat angular distribution as expected. A track length threshold of about $120 \mathrm{~nm}$ was derived with this approach.

\section{Background}

Background sources for dark matter searches are $\alpha$ and $\beta$ particles, $\gamma$-rays and neutron induced 

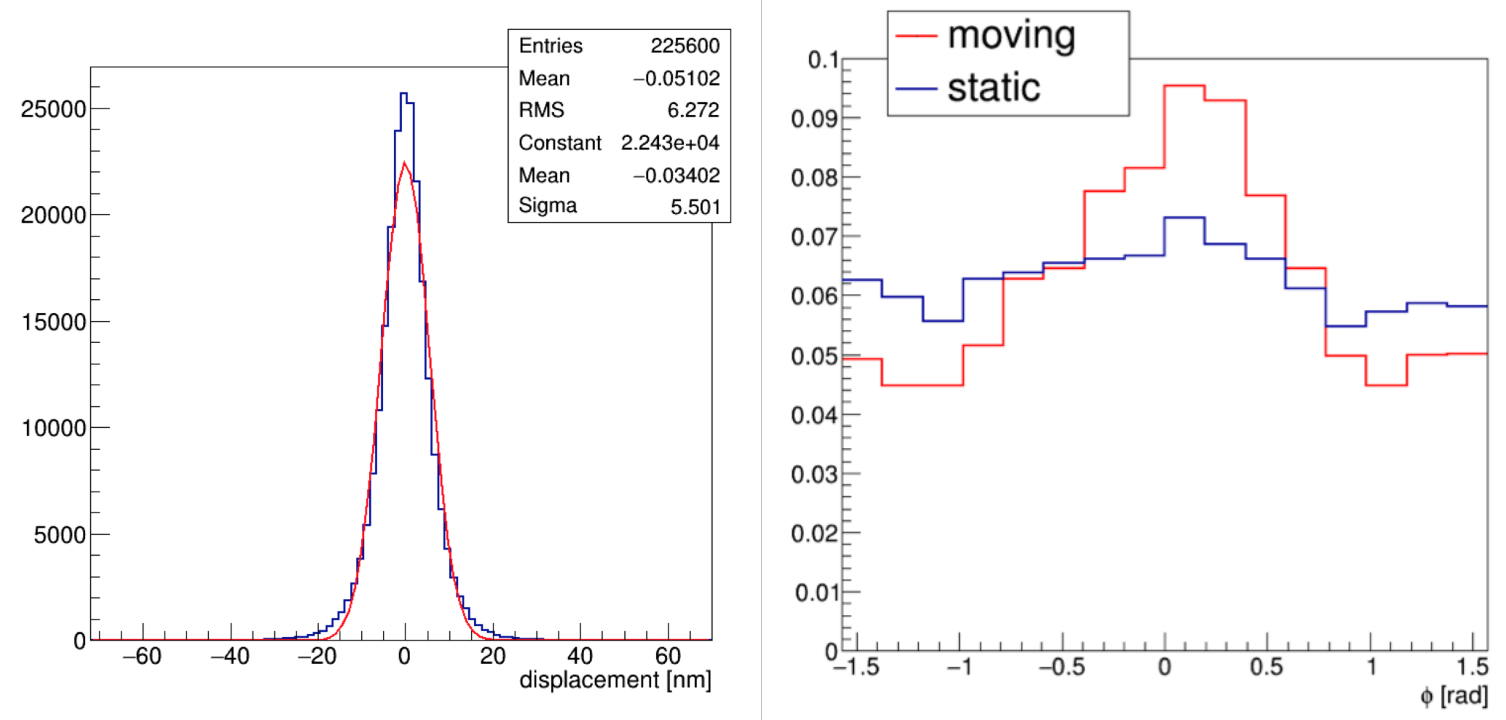

Figure 1: a) Position accuracy of about $6 \mathrm{~nm}$ with the resonant light scattering for $10 \mathrm{keV}$ C-ions; b) $2 \mathrm{D}$ angular distribution obtained by the direction of the barycentre shift for moving (red) and static (blue) grains for $60 \mathrm{keV} \mathrm{C}$-ions.

recoils, while NIT are essentially not sensitive to minimum ionizing particles (MIP).

The $\alpha$-particles, produced by $\mathrm{U}$ and Th radioactive chains and Radon, can be discarded by an upper cut on the track length. The $\beta$-rays produced in $14 \mathrm{C}$ decay could be rejected by properly regulating the emulsion response in terms of number of sensitized crystals per unit path length (i.e. the sensitivity), or through a chemical treatment of the emulsion itself and exploiting the response of $\beta$-rays to the polarized light scattering or performing a cryogenic exposure and by exploiting the phonon effect.

Neutron induced recoils are the main background source because they are not distinguishable from the expected WIMP signal, except for the isotropic angular distribution and for the typical track length, largely exceeding the range expected for WIMP-induced recoils. Three types of neutron sources affect underground experiments: radiogenic neutrons in the $\mathrm{MeV}$ range produced in $(\alpha, \mathrm{n})$ and spontaneous fission reactions in the detector due to its intrinsic radioactive contaminants, cosmogenic neutrons with energy spectrum extending to $\mathrm{GeV}$ energies induced by muons penetrating underground through the rock, neutrons induced by environmental radioactivity. External neutron flux can be reduced to a reasonable level with an appropriate shielding. The intrinsic emulsion radioactivity, instead, has been evalutated by using a dedicated MC simulation based on the SOURCES code [15] and a GEANT4-based simulation. The detectable neutron-induced background would be $0.06 \mathrm{n} / \mathrm{yr} / \mathrm{kg}$ [16].

\section{Technical test and pilot experiment}

A technical test with the exposure of $10 \mathrm{~g}$ emulsions has been performed in June 2019 the Gran Sasso Underground Laboratory (Fig. 2). The analysis of the emulsion films is on-going, in parallel 
with the construction of the underground infrastructure for emulsion production and development and the studies of the final detector design.

A pilot experiment will be performed with the exposure of $10 \mathrm{~kg}$ mass target on a time scale of one year with the aim of measuring the detectable background from environmental and intrinsic sources and to validate estimates from measurements [16] and simulations. The confirmation of a negligible background will pave the way for the construction of a pilot experiment with an exposure on the $\sim 10 \mathrm{~kg}$ year scale, which will act as a demonstrator to further extend the sensitivity towards the neutrino floor.
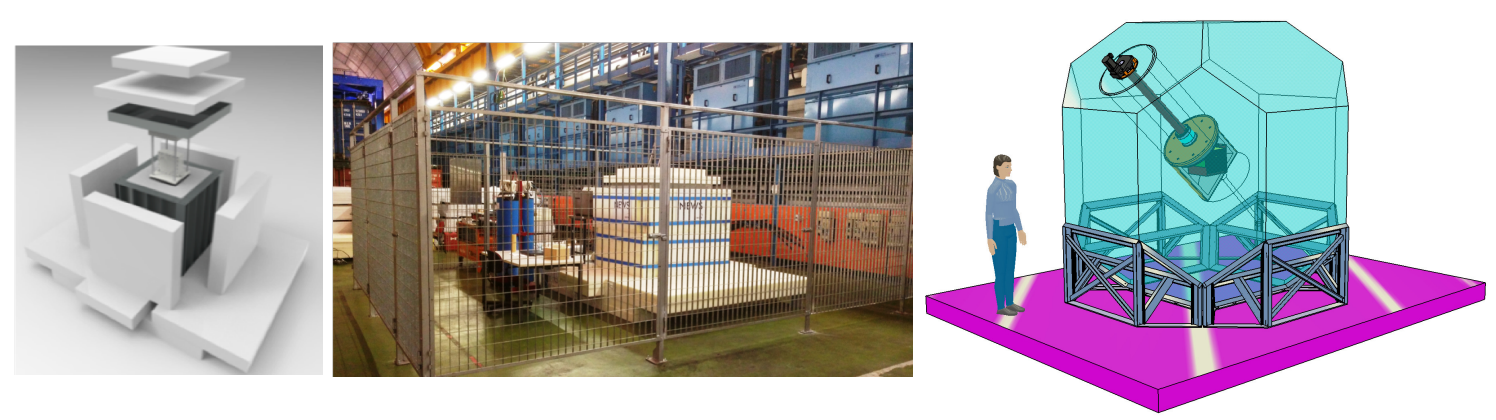

Figure 2: a) Shield exploded view; b) a picture of the detector installed in Hall B at LNGS; c) A scheme of the detector for the pilot experiment

\section{Conclusion}

The NEWSdm experiment is meant to be the first experiment with a solid target for directional dark matter searches. Its detector idea, based on a novel kind of nuclear emulsions and readout tecnology, allows to extend dark matter searches beyond the neutrino floor and provide an unambiguous signature of the detection of Galactic dark matter.

In Figure 3 the $90 \%$ C.L. upper limit, in case of null observation for an exposure of $10 \mathrm{~kg} \times$ year of NIT emulsions, with a minimum detectable track length ranging from $200 \mathrm{~nm}$ down to 50 $\mathrm{nm}$ and in the hypothesis of zero background, is shown. Even not including the directionality discrimination of the signal and assuming to reach a negligible background level, such an experiment would cover a large part of the parameter space indicated by the DAMA/ LIBRA results with a small detector mass, using a powerful and complementary approach.

\section{References}

[1] PLAnCK collaboration, N. Aghanim et al., Planck 2015 results. XI. CMB power spectra, likelihoods, and robustness of parameters, Astron. Astrophys. 594 (2016) A11, [1507.02704].

[2] D. N. Spergel, The Motion of the Earth and the Detection of Wimps, Phys. Rev. D37 (1988) 1353.

[3] C. E. Aalseth et al., Search for an Annual Modulation in a P-type Point Contact Germanium Dark Matter Detector, Phys. Rev. Lett. 107 (2011) 141301, [1106.0650].

[4] J. B. R. Battat et al., Readout technologies for directional WIMP Dark Matter detection, Phys. Rept. 662 (2016) 1-46, [1610.02396]. 


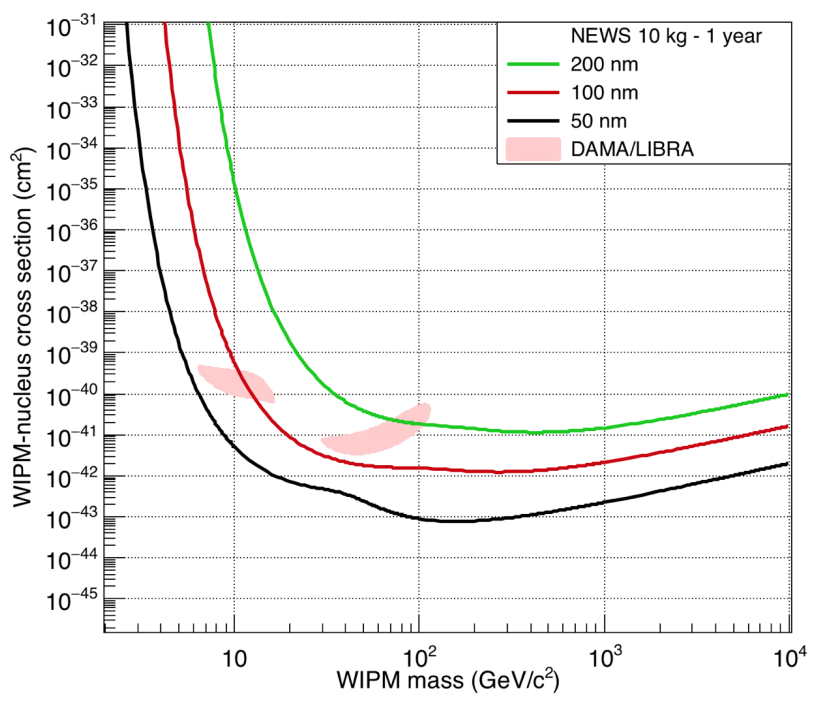

Figure 3: The $90 \%$ C.L. upper limits for a NIT detector with an exposure of $1 \mathrm{~kg} \times$ year, a threshold ranging from $200 \mathrm{~nm}$ down to $50 \mathrm{~nm}$, in the zero background hypothesis. The directionality information is not included

[5] NEWS collaboration, A. Aleksandrov et al., NEWS: Nuclear Emulsions for WIMP Search, 1604.04199.

[6] M. Natsume, K. Hoshino, M. Nakamura, T. Nakano, K. Niwa, O. Sato et al., Low-velocity ion tracks in fine grain emulsion, Nucl. Instrum. Meth. A575 (2007) 439-443.

[7] T. Naka et al., Fine grained nuclear emulsion for higher resolution tracking detector, Nucl. Instrum. Meth. A718 (2013) 519-521.

[8] R. Acquafredda et al., The OPERA experiment in the CERN to Gran Sasso neutrino beam, JINST 4 (2009) P04018.

[9] A. Alexandrov, V. Tioukov and M. Vladymyrov, Further progress for a fast scanning of nuclear emulsions with Large Angle Scanning System, JINST 9 (2014) C02034.

[10] N. Armenise et al., High-speed particle tracking in nuclear emulsion by last-generation automatic microscopes, Nucl. Instrum. Meth. A551 (2005) 261-270.

[11] K. Morishima and T. Nakano, Development of a new automatic nuclear emulsion scanning system, S-UTS, with continuous 3D tomographic image read-out, JINST 5 (2010) P04011.

[12] S. Aoki, K. Hoshino, M. Nakamura, K. Niu, K. Niwa and N. Torii, The Fully Automated Emulsion Analysis System, Nucl. Instrum. Meth. B51 (1990) 466-472.

[13] M. Kimura and T. Naka, Submicron track readout in fine-grained nuclear emulsions using optical microscopy, Nucl. Instrum. Meth. A680 (2012) 12-17.

[14] H. Tamaru, H. Kuwata, H. T. Miyazaki and K. Miyano, Resonant light scattering from individual ag nanoparticles and particle pairs, Applied Physics Letters 80 (2002) 1826-1828, [https://doi.org/10.1063/1.1461072].

[15] W. Wilson et al., Sources 4a: A code for calculating (alpha,n), spontaneous fission, and delayed neutron sources and spectra, . 
[16] A. Alexandrov et al., Intrinsic neutron background of nuclear emulsions for directional Dark Matter searches, Astropart. Phys. 80 (2016) 16-21, [1507. 03532]. 